\title{
A proof of Erdős-Fishburn's conjecture for $g(6)=13$
}

\author{
Xianglin Wei* \\ College of Science \\ Hebei University of Science and Technology \\ Hebei Province, China \\ wxlhebtu@126.com
}

Submitted: Mar 1, 2011; Accepted: Nov 20, 2012; Published: Dec 6, 2012

Mathematics Subject Classifications: 52B05, 52C10

\begin{abstract}
A planar point set $X$ in the Euclidean plane is called a $k$-distance set if there are exactly $k$ distances between two distinct points in $X$. An interesting problem is to find the largest possible cardinality of $k$-distance sets. This problem was introduced by Erdős and Fishburn (1996). Maximum planar sets that determine $k$ distances for $k$ less than 5 has been identified. The 6-distance conjecture of Erdös and Fishburn states that 13 is the maximum number of points in the plane that determine exactly 6 different distances. In this paper, we prove the conjecture.
\end{abstract}

Keywords: 6-distance conjecture; Diameter graph; Independent set.

\section{Introduction}

A planar point set $X$ in Euclidean plane is called a $k$-distance set if there are exactly $k$ different distances between two distinct points in $X$. For two point sets, we say that they are isomorphic if there exists a similar transformation from one to the other. One of the many interesting problems on $k$-distance sets is to determine the largest possible cardinality of $k$-distance sets and to classify these $k$-distance sets. We denote this number by $g(k)$. A $k$-distance set $X$ is said to be maximum if $X$ has $g(k)$ points. Let $R_{n}$ denote the vertices of a regular $n$-gon, $R_{n}^{+}$be $R_{n}$ plus its center, $R_{n}-i$ denote a set of $n-i$ vertices of $R_{n}$. When $i \geqslant 2, R_{n}-i$ has dissimilar versions depending on which $i$ points of $R_{n}$ are absent. Clearly $g(k) \geqslant 2 k+1$ since $R_{2 k+1}$ is a $k$-distance set. The triangular

\footnotetext{
*Supported by National Natural Science Foundation of China (10901045, 11171088), NSF of Hebei (A2010000828), SF of Hebei University of Science and Technology (QD200955).
} 
lattice $L_{\triangle}=\left\{a(1,0)+b\left(\frac{1}{2}, \frac{\sqrt{3}}{2}\right): a, b \in \mathbb{Z}\right\}$. Erdös-Fishburn [1] presented the following two conjectures:

Conjecture 1. [1] $g(6)=13$, and this is realized only by $R_{13}$, or $R_{12}^{+}$, or the 13 -point subset of Figure 1.

Conjecture 2. [1] For every $k \geqslant 3$, some $g(k)$-point set of the triangular lattice $L_{\triangle}$ has exactly $k$ interpoint distances. Moreover, if $k \geqslant 7$, every $g(k)$-point subset of the plane that determines $k$ different distances is similar to a subset of $L_{\triangle}$.

In [1] the author determined $g(k)$ for $k \leqslant 5$ and classified maximum $k$-distance sets for $k \leqslant 4$, recall that $g(1)=3$ which is realized by $R_{3}, g(2)=5$ and every 5 -point two-distance set is isomorphic to $R_{5}, g(3)=7$ and every 7 -point three-distance set is isomorphic to $R_{7}$ or $R_{6}^{+}, g(4)=9$, and there exists 9-point subset of $L_{\triangle}$ with 4-distance. Shinohara [5] proved that 12-point 5-distance set which realized for $g(5)=12$ is unique up to similar transformation, that is a subset of $L_{\triangle}$. Shinohara [6] classified 3-distance sets with at least five points. In this note we prove that any 14-point set determines at least 7 distances, that is to say, the conjecture $g(6)=13$ is correct.

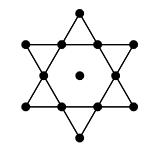

Figure 1: 13-point 6-distance set configuration in $L_{\triangle}$.

\section{Preliminaries and Related Lemmas}

As in [7] we recall some notations. Let $d(x, y)$ denote the distance between two planar points $x$ and $y$. Let $D=D(X)$ be the diameter of a finite set $X$, and let $X_{D}=\{x \in$ $X: d(x, y)=D$ for some $y \in X\}$ and $m=m(X)=\left|X_{D}\right|$. The diameter graph $D G(X)$ of $X$ is the graph with $X$ as its vertices and where two vertices $x, y \in X$ are adjacent if $d(x, y)=D$. We denote a path and a cycle with $n$ vertices by $P_{n}$ and $C_{n}$, respectively. For $v \in X_{D}$, let $d(v)$ denote the number of $D$-lengths segments connected with $v$ in $D G\left(X_{D}\right)$. Clearly $D G\left(X_{D}\right)$ has no isolated vertex, and every two $D$-length segments in $D G\left(X_{D}\right)$ must cross if they do not share an end point.

We say a point set $P$ is convex when it forms the vertex set of a point set. When indexing a set of $t$ points, we identify indices modulo $t$. As in [5] we denote the set of all $n$-point $k$-distance sets and the set of all $n$-point convex $k$-distance sets by $E_{n}(k)$ and $M_{n}(k)$, respectively. Let $X_{D}=\{1,2,3, \ldots, m\}$, here $m$ points are consecutive in counterclockwise order, we say segment $[i, i+1]$ be an edge of $X_{D}$ for every $i \in X_{D}$. A subset $H$ of $V(G)$ is an independent set of $V(G)$ if no two vertices in $\mathrm{H}$ are adjacent, and $\mathrm{H}$ is said to be maximal if no other independent sets contain $H$, the independence number $\alpha(G)$ of a graph $G$ is the maximum cardinality among the independent sets of $G$. In the following some proofs are omitted because of the restriction of the length of the paper. 
Lemma 3. [1] Let $D$ be the diameter of an n-point planar set $X$ with $n \geqslant 3$ and $m=\left|X_{D}\right|$. Then (a) if $m \geqslant 3$, the points in $X_{D}$ are the vertices of a convex $m$-gon;

(b) $D$ can be eliminated as an interpoint distance by removing at most $\left\lceil\frac{m}{2}\right\rceil$ points from $X$, where $\left\lceil\frac{m}{2}\right\rceil$ is the smallest integer at least $m / 2$.

Lemma 4. [3] [4] Suppose $S$ is the vertex set of a convex $n$-gon, $n \geqslant 3$, that determines exactly $t$ different distances. Then $t \geqslant\lfloor n / 2\rfloor$. Moreover:

(i) if $n$ is odd and $t=(n-1) / 2, S$ is $R_{n}$;

(ii) if $n$ is even, $t=n / 2$, and $n \geqslant 8, S$ is $R_{n}$ or $R_{n+1}-1$;

(iii) if $(n, t)=(7,4), S$ is $R_{8}-1$ or $R_{9}-2$;

(iv) if $(n, t)=(9,5), S$ is $R_{10}-1$ or $R_{11}-2$;

Lemma 5. [5] Let $G=D G(X)$ for $X$. Then

(a) $G$ contains no $C_{2 k}$ for any $k \geqslant 2$;

(b) if $G$ contains $C_{2 k+1}$, then any two vertices in $V(G) \backslash V\left(C_{2 k+1}\right)$ are not adjacent and every vertex not in the cycle is adjacent to at most one vertex of the cycle, where $V(G)$ denote the vertex set of the simple graph $G$. In particular, $G$ contains at most one cycle.

Lemma 6. [7] If $d(v)=k \geqslant 2$ for $v \in X_{D}$ in the diameter graph $D G\left(X_{D}\right)$ of $X_{D}$, then the $k$ vertices having $D$-length with $v$ are consecutive.

Lemma 7. [7] For a planar point set $X$ with $m=\left|X_{D}\right|$, let $X_{D}=\{1,2,3, \ldots, m\}$, $m$ points are consecutive with counter-clockwise order. If for a subset $S \subset X_{D}, S=$ $\{k, k+1, k+2, \ldots, k+l-1\}$, the segment $[k, k+l-1]$ is the max-length segment of $S$ and $d(k, k+i)<d(k, k+l-1)$ for any $i=1,2,3, \ldots, l-2$, then $d(k, k+1)<d(k, k+2)<$ $d(k, k+3)<\ldots<d(k, k+l-1) \leqslant D$.

Lemma 8. [2] Let $X$ be the vertex set of convex $n$-gon with $s$ intervertex distances $d_{1}>$ $d_{2}>\cdots>d_{s}$. If a side of the convex $n$-gon has the $d_{1}$-length, then $s \geqslant n-2$.

Lemma 9. [5] Let $G=D G(X)$ be the diameter graph of $X$ with $|X|=n$. If $G \neq C_{n}$, then we have $\alpha(G) \geqslant\lceil n / 2\rceil$.

\section{Classification of 10 point 5-distance}

Lemma 10. Let $X$ be a 10-point 5-distance set. Then $X$ contains a subset $Y \in$ $\left\{R_{6}^{+}, R_{7}, R_{8}, R_{9}-1, R_{10}-2, R_{11}-3\right\} \bigcup E_{7}(4)$.

Proof. Let $X$ be a 10-point 5-distance set, and $m=\left|X_{D}\right|$. If $m \geqslant 9$, then by Lemma 3 and Lemma 4, the subset $X_{D} \subset X$ is a convex $m$-gon, and $X$ contains a subset $Y \in$ $\bigcup_{k \leqslant 5} M_{9}(k)=\left\{R_{9}, R_{10}-1, R_{11}-2\right\}$. When $m=8$, the proof is as showed in Lemma 7 and Lemma 8 in [7], $X$ contains a subset $Y \in \bigcup_{k \leqslant 4} E_{7}(k) \bigcup\left\{R_{8}, R_{9}-1, R_{10}-2, R_{11}-3\right\}$. Recall that $g(3)=7$ and $E_{7}(3)=\left\{R_{6}^{+}, R_{7}\right\}, \bigcup_{k \leqslant 4} E_{7}(k)=\left\{R_{6}^{+}, R_{7}\right\} \bigcup E_{7}(4)$. When $m=7$, and if $D G\left(X_{D}\right)=C_{7}$, we have showed $X_{D}=R_{7}$ as in [9]; and if $D G\left(X_{D}\right) \neq C_{7}$, by Lemma $9, \alpha\left(D G\left(X_{D}\right)\right) \geqslant 4, X$ contains a subset $Y \in \bigcup_{k \leqslant 4} E_{7}(k)$ by removing three points from $X_{D}$ with the diameter $D$ been eliminated. If $m \leqslant 6$, then $10-\left\lceil\frac{m}{2}\right\rceil \geqslant 7$, and by Lemma $3, X$ contains a subset $Y \in \bigcup_{k \leqslant 4} E_{7}(k)$ too. 
Theorem 11. Every 10-point 5-distance set in the plane is isomorphic to $R_{9}^{+}, R_{10}, R_{11}-1$, double $R_{5}$ with the same center as shown in Figure 2q, or one of the sixteen 10-point configurations in $L_{\triangle}$ as shown in Figure 2a-2p.

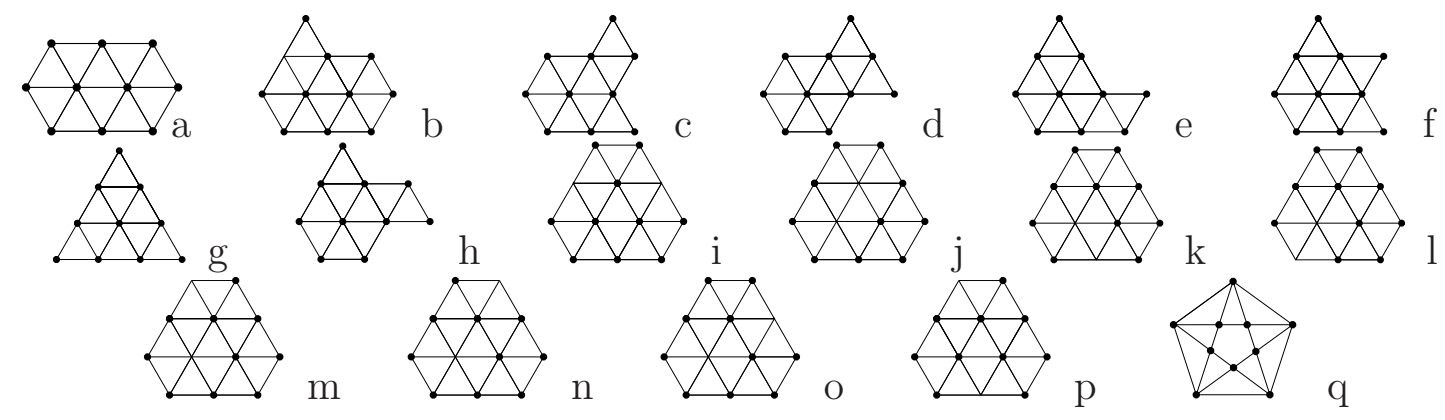

Figure 2: 10-point 5-distance sets.

Proof. By Lemma 10, $X$ contains a subset $Y \in\left\{R_{6}^{+}, R_{7}, R_{8}, R_{9}-1, R_{10}-2, R_{11}-\right.$ $3\} \bigcup E_{7}(4)$. When $X$ contains a subset $Y \in\left\{R_{7}, R_{8}\right\}$, clearly they can not be extended to a 10-point 5-distance set. When $X$ contains a subset $Y \in\left\{R_{9}-1, R_{10}-2, R_{11}-3\right\}$, it is easy to see that $X$ can be the 10 -point 5 -distance sets $R_{9}^{+}, R_{10}, R_{11}-1$. When $X$ contains $R_{6}^{+}$, clearly $X \subset L_{\triangle}$ and $X$ can be one of the eight 10-point 5-distance sets as shown in Figure 2a-2h. Now assume $X$ contains a subset $Y \in E_{7}(4)$ given in [8]. Using elementary plane geometry we can easily obtain nine more 10 -point 5 -distance sets as shown in Figure 2i-2q.

\section{No 14-point 6-distance set with $m=11$}

Lemma 12. Let $X$ be a 14-point 6-distance set and $m=\left|X_{D}\right|=11$. Then for every point $v \in D G\left(X_{D}\right), d(v) \leqslant 2$.

Proof. Let $X$ be a 14-point 6-distance set, and 6 distances are $D=d_{1}>d_{2}>d_{3}>d_{4}>$ $d_{5}>d_{6}$. By lemma 3 , we know $X_{D}$ is a convex set. Let $X_{D}=\{1,2,3, \ldots, 11\}, 11$ points are consecutive with counter-clockwise order. If $d(i, i+1)=D$ or $d(i, i+2)=D$ or $d(i, i+3)=D$, then by lemma $8, X_{D}$ determines at least 7 distances, a contradiction to 6 -distance. So in the following we may assume for any $i \in X_{D}, d(i) \leqslant 4$, and $d(i, j) \neq D$, where $j=i+1, i+2, i+3$. If for $d(i)=3$ or 4 we obtain a contradiction to 6 -distance, then the result is correct.

At first assume there exists $i$ such that $d(i)=4$. Without loss of generality we may assume $d(1)=4$. Then clearly $d(1,5)=d(1,6)=d(1,7)=d(1,8)=D$. It is easy to see $d(2,7) \neq D$ since $d(1,8)=D$, and by Lemma $7, d_{6}=d(6,7)<d(5,7)<$ $d(4,7)<d(3,7)<d(2,7)<d(1,7)=D$, and so $d(4,5)=d(5,6)=d(6,7)=d_{6}$ and $d(4,6)=d(5,7)=d_{5}, \triangle 456 \cong \triangle 567$ which contradicts the fact $\angle 456 \neq \angle 567$. Secondly assume $d(i) \leqslant 3$ for every $i \in X_{D}$. Similar as before we may assume $d(1)=3$ and 
$d(1,6)=d(1,7)=d(1,8)=D$. By Lemma $7, d_{6}=d(6,7)<d(5,7)<d(4,7)<d(3,7)<$ $d(2,7)<d(1,7)=D, d(2,3)=d(3,4)=d(4,5)=d(5,6)=d(6,7)=d_{6}$ and $d(2,4)=$ $d(3,5)=d(4,6)=d(5,7)=d_{5}, d(2,5)=d(3,6)=d(4,7)=d_{4}, d(2,6)=d(3,7)=d_{3}$. It follows that points $2,3,4,5,6,7$ lie on a circle. If $d(3,8) \neq D$, then by Lemma 6 and Lemma $7, d_{6}=d(7,8)<d(6,8)<d(5,8)<d(4,8)<d(3,8)<d(2,8)=D$, and points $2,3,4,5,6,7,8$ lie on a circle, that is to say, $d(1,5)=D$ since $d(1,6)=$ $d(1,7)=d(1,8)=D$, a contradiction. So $d(3,8)=D$, and then $d(2,8)=D$. Clearly $d(1,2) \leqslant d_{5}, d(7,8) \leqslant d_{5}$. If $d(7,8)=d_{5}$, then by Lemma $7, d(6,8)=d(4,7)=d_{4}$ and $d(7,8)=d(4,6)=d_{5}$, and points $4,6,7,8$ lie on a circle, that is to say, $d(1,4)=D$ since $d(1,6)=d(1,7)=d(1,8)=D$, a contradiction. So $d(7,8)=d_{6}$, similarly $d(1,2)=d_{6}$. If $d(1,3)=d_{5}$, then points $1,2,3,4$ lie on a circle, that is to say, $d(4,8)=D$ since $d(1,8)=d(2,8)=d(3,8)=D$, a contradiction. So $d(1,3)=d_{4}$, similarly $d(6,8)=d_{4}$. Finally by Lemma $7, d_{4}=d(1,3)<d(1,4)<d(1,5)<d(1,6)=D$, points $1,2,7$, 8 lie on a circle, points 1,3,4, 7 lie on a circle, and so points 1, 2, 3, 4, 5, 6, 7, 8 lie on the circle, a contradiction.

Theorem 13. Let $X$ be a 14-point 6-distance set and $m=\left|X_{D}\right|=11$. Then $X$ contains a subset $R_{11}$ or $R_{13}-2$.

Proof. Clearly $d(v) \leqslant 2$ for every point $v \in D G\left(X_{D}\right)$ by Lemma 12 . Recall that $D G\left(X_{D}\right)$ has no isolated vertex. If $D G\left(X_{D}\right) \neq C_{11}$ but contains a cycle $C_{2 k+1}$, by Lemma 5 we know the remaining points in $V(G) \backslash V\left(C_{2 k+1}\right)$ must connect with the points on the cycle, thus there exists a point $v \in D G\left(X_{D}\right), d(v) \geqslant 3$, a contradiction. Then we conclude $D G\left(X_{D}\right)$ can be $C_{11}, P_{11}, P_{9} \cup P_{2}, P_{8} \cup P_{3}, P_{7} \cup P_{4}, P_{7} \cup 2 P_{2}, P_{6} \cup P_{5}, P_{6} \cup P_{3} \cup P_{2}$, $P_{5} \bigcup P_{4} \bigcup P_{2}, P_{5} \bigcup 2 P_{3}, P_{5} \bigcup 3 P_{2}, 2 P_{4} \bigcup P_{3}, P_{4} \bigcup P_{3} \bigcup 2 P_{2}, 3 P_{3} \bigcup P_{2}$ or $P_{3} \bigcup 4 P_{2}$.

By lemma 4 , we know $X_{D}$ is a convex set. Let $X_{D}=\{1,2,3, \ldots, 11\}, 11$ points are consecutive and always in counter-clockwise order. Since $g(4)=9, X_{D}$ has at least 5 distinct distances. If $X_{D}$ is a 5 -distance set, then by lemma 3 (i), $X_{D}=R_{11}$. So in the following we need to consider the case that $X_{D}$ is a 6 -distance set, and 6 distances are $D=d_{1}>d_{2}>d_{3}>d_{4}>d_{5}>d_{6}$. We depart three parts to prove.
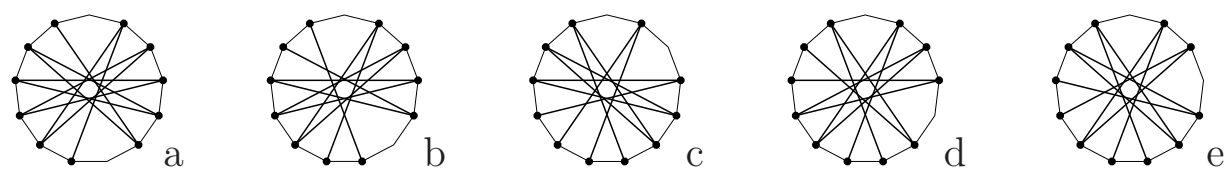

Figure 3: Dissimilar versions of $R_{13}-2$.

Part I: Suppose $D G\left(X_{D}\right)=P_{11}$. By Lemma 6 we may denote $d(1,7)=d(2,7)=$ $d(2,8)=d(3,8)=d(3,9)=d(4,9)=d(4,10)=d(5,10)=d(5,11)=d(6,11)=D$. Ву Lemma $7, d(i, i+1)=d_{6}$ for $i=1,2,3,4,5$, and $d_{6} \leqslant d(i, i+1) \leqslant d_{5}$ for $i=6,7,8,9,10,11$. Clearly points $1,2,3,4,5,6$, lie on a circle, points $4,6,10,11$ lie on a circle, points 3, 5, 9, 10 lie on a circle, points 2, 4, 8, 9 lie on a circle, points 1, 3, 7, 8 lie on a circle. If $d(7,8)=d_{5}$, then $d_{5}=d(7,8)<d(6,8)<d(5,8)<d(4,8)<d(3,8)=D$, points 2, 5, 6, 8 lie on a circle, and so points 1, 2, 7, 8 lie on a circle, which implies 
$d_{6}=d(1,2)=d(7,8)=d_{5}$, a contradiction. So $d(7,8)=d_{6}$, by the similar reason $d(8,9)=d(9,10)=d(10,11)=d_{6}$. Now we conclude all points of $X_{D}$ lie on a circle, and $d(1,11)=d(6,7)=d_{5}, X_{D}=R_{13}-2$ as shown in Figure 3 a.

Suppose $D G\left(X_{D}\right)=P_{9} \cup P_{2}$. By Lemma 6 we may denote $d(1,7)=d(2,8)=d(3,8)=$ $d(3,9)=d(4,9)=d(4,10)=d(5,10)=d(5,11)=d(6,11)=D$. By Lemma $7, d(i, i+$ $1)=d_{6}$ for $i=1,2,3,4,5,6$, and $d_{6} \leqslant d(i, i+1) \leqslant d_{5}$ for $i=7,8,9,10,11$. Clearly points 1, 2, 3, 4, 5, 6, 7 lie on a circle, points 2, 4, 8, 9 lie on a circle, points 3, 5, 9, 10 lie on a circle, points $4,6,10,11$ lie on a circle. If $d(8,9)=d_{5}$, then $d_{5}=d(8,9)<d(7,9)<$ $d(6,9)<d(5,9)<d(4,9)=D$, points $3,6,7,9$ lie on a circle, and so points $2,3,8,9$ lie on a circle, which implies $d_{6}=d(2,3)=d(8,9)=d_{5}$, a contradiction. So $d(8,9)=d_{6}$, by the similar reason $d(9,10)=d(10,11)=d_{6}$. Now all points of $X_{D}$ lie on a circle, and $d(1,11)=d(7,8)=d_{5}, X_{D}=R_{13}-2$ as shown in Figure $3 \mathrm{~b}$.

By the similar way, when $D G\left(X_{D}\right)=P_{8} \cup P_{3}, P_{7} \cup P_{4}$, or $P_{6} \cup P_{5}$, we can always prove that all points of $X_{D}$ lie on a circle, and $X_{D}=R_{13}-2$ as shown in Figure 3c-3e.

Part II: Suppose $D G\left(X_{D}\right)=P_{7} \bigcup 2 P_{2}$. By Lemma 6 we may denote $d(1,6)=d(2,7)=$ $d(3,8)=d(3,9)=d(4,9)=d(4,10)=d(5,10)=d(5,11)=D$. By Lemma $7, d(i, i+1)=$ $d_{6}$ for $i=1,6,7,8,9,10,11$, and $d_{6} \leqslant d(i, i+1) \leqslant d_{5}$ for $i=2,3,4,5$. Clearly points $1,2,6$, $7,8,9,10,11$ lie on a circle, points $3,4,8,10$ lie on a circle, points 4, 5, 9, 11 lie on a circle. If $d(3,4)=d_{5}$, then $d_{5}=d(3,4)<d(2,4)<d(1,4)<d(4,11)<d(4,10)=D$, points 1 , 4, 7, 11 lie on a circle, and so conclude points 3, 4, 9, 10 lie on a circle, which implies $d_{5}=d(3,4)=d(9,10)=d_{6}$, a contradiction. So $d(3,4)=d_{6}$, similarly $d(4,5)=d_{6}$. Now we conclude all points of $X_{D}$ lie on a circle, $\triangle 126 \cong \triangle 893$ or $\triangle 1 * 6 \cong \triangle 893$ where $*=11$, but implies $D>d(2,6)=d(3,9)=D$ or $D>d(6,11)=d(3,9)=D$, a contradiction.

When $D G\left(X_{D}\right)=P_{6} \cup P_{3} \cup P_{2}, P_{5} \cup P_{4} \cup P_{2}, P_{5} \cup 2 P_{3}, P_{5} \cup 3 P_{2}, 2 P_{4} \cup P_{3}, 3 P_{3} \cup P_{2}$, or $P_{3} \cup 4 P_{2}, P_{4} \cup P_{3} \cup 2 P_{2}$, we can always conclude all points of $X_{D}$ lie on a circle, and then find a contradiction, that is to say, these types are not realizable for 6-distance.

Part III: Suppose $D G\left(X_{D}\right)=C_{11}$. By Lemma 7 , we can see that $d(x, x+1) \leqslant d_{5}$ for any $x \in X_{D}$. If all edges of $X_{D}$ have the same length, then clearly all points of $X_{D}$ lie on a circle, and hence $X_{D}=R_{11}$, which is not 6-distance. So there exist two consecutive edges which have different lengths. Without loss of generality, we may assume $d(1,2)=d_{5}$ and $d(2,3)=d_{6}$. By Lemma $7, d_{5}=d(1,2)<d(2,11)<d(2,10)<d(2,9)<d(2,8)=D$, $d_{5}=d(1,2)<d(1,3)<d(1,4)<d(1,5)<d(1,6)=D$.

Assume $d(3,4)=d_{5}$. Then $d_{4}=d(2,4)$ by Lemma 7 , and points $1,2,3,4$ lie on a circle. If $d(1,11)=d_{5}$, then points $1,3,4,11$ lie on a circle, and hence points $1,2,3,11$ lie on a circle, which implies $d_{6}=d(2,3)=d(1,11)=d_{5}$, a contradiction. If $d(1,11)=d_{6}$, then points 1, 2, 3, 11 lie on a circle, and hence points 1, 3, 4, 11 lie on a circle, which implies $d_{5}=d(3,4)=d(1,11)=d_{6}$, a contradiction. So $d(3,4)=d_{6}$.

Assume $d(4,5)=d_{5}$. Then $d_{4}=d(4,6)=d(3,5), d_{3}=d(3,6)$ by Lemma 7 , and points $1,2,4,5$ lie on a circle, points $1,3,4,6$ lie on a circle. If $d(5,6)=d_{5}$, then points 1, 2, 5, 6 lie on a circle, and so points 2, 3, 5, 6 lie on a circle, which implies $d_{6}=d(2,3)=d(5,6)=d_{5}$, a contradiction. If $d(5,6)=d_{6}$, then points $3,4,5,6$ lie on a circle, and so points $1,2,5,6$ lie on a circle, which implies $d_{6}=d(5,6)=d(1,2)=d_{5}$, a contradiction. So $d(4,5)=d_{6}$. 
By the similar way we can also prove $d(5,6)=d(6,7)=d(7,8)=d(8,9)=d(9,10)=$ $d(10,11)=d_{6}$. Then points $2,3,4,5,6,7,8,9,10,11$ lie on a circle, and $d_{5} \leqslant$ $d(6,8) \leqslant d_{4}$. When $d(6,8)=d_{5}$, points $1,2,6,8$ lie on a circle; when $d(6,8)=d_{4}$, points 1, 3, 6, 8 lie on a circle. So conclude all points of $X_{D}$ lie on the circle, which implies $d_{6}=d(6,7)=d(1,2)=d_{5}$, a contradiction. That is to say, $D G\left(X_{D}\right)=C_{11}$ is not realizable for 6 -distance.

Theorem 14. No 14-point 6-distance set with $m=\left|X_{D}\right|=11$ exists.

Proof. If $X$ is a 14-point 6-distance set with $m=\left|X_{D}\right|=11$, then by Theorem 13, $X$ contains a subset $Y \in\left\{R_{11}, R_{13}-2\right\}$. Adding points to $R_{11}$ or $R_{13}-2$ such that the total number of points being 14 always force a seventh distance, which contradicts 6-distance.

\section{$5 \quad$ No 14-point 6-distance set with $m=10$}

Lemma 15. Let $X$ be a 14-point 6-distance set and $m=\left|X_{D}\right|=10$. Then $X$ contains a subset $Y \in E_{10}(5)$, or for every point $v \in X_{D}, d(v) \leqslant 2$.

Proof. Let $X$ be a 14-point 6-distance set, and 6 distances are $D=d_{1}>d_{2}>d_{3}>d_{4}>$ $d_{5}>d_{6}$. Let $X_{D}=\{1,2,3, \ldots, 10\}, 10$ points are consecutive with counter-clockwise order. By lemma 3, we know $X_{D}$ is a convex set. If $d(i, i+1)=D$ or $d(i, i+2)=D$, then by lemma $8, X_{D}$ determines at least 7 distances, a contradiction to 6 -distance. If $d(i, i+3)=D$, then we know $X$ contains a subset $Y \in E_{10}(5)$ by removing four points $i, i+1, i+2, i+3$ from $X_{D}$ with the diameter $D$ been eliminated, since two $D$-length segments in $X_{D}$ must cross if they do not share an end point. So in the following we may assume for any $i \in X_{D}, d(i) \leqslant 3$, and $d(i, j) \neq D$, where $j=i+1, i+2, i+3$. If for any $d(i)=3$ we obtain a contradiction to 6 -distance or prove that $X$ contains a subset $Y \in E_{10}(5)$, then the result is correct. Now without loss of generality we may assume $d(1)=3$, and $d(1,5)=d(1,6)=d(1,7)=D$.

If $d(2,7) \neq D$, then we know $X$ contains a subset $Y \in E_{10}(5)$ by removing four points $1,8,9,10$ from $X_{D}$ with the diameter $D$ been eliminated. So $d(2,7)=D$, similarly $d(5,10)=D$. We can prove the result is correct by the similar way as in the proof of Lemma 12.

Theorem 16. Let $X$ be a 14-point 6-distance set and $m=\left|X_{D}\right|=10$. Then $X$ contains a subset $Y \in\left\{R_{12}-2, R_{13}-3\right\} \bigcup E_{10}(5)$.

Proof. By Lemma 15, we know $X$ contains $E_{10}(5)$, or for every point $v \in X_{D}, d(v) \leqslant 2$. As in Theorem 13 we know $D G\left(X_{D}\right)$ can be $P_{10}, P_{8} \cup P_{2}, P_{7} \bigcup P_{3}, P_{6} \bigcup P_{4}, P_{6} \bigcup 2 P_{2}$, $2 P_{5}, P_{5} \bigcup P_{3} \bigcup P_{2}, 2 P_{4} \bigcup P_{2}, P_{4} \bigcup 2 P_{3}, P_{4} \bigcup 3 P_{2}, 2 P_{3} \bigcup 2 P_{2}$ or $5 P_{2}$. If $X_{D}$ is a 5 -distance set, by lemma $4, X_{D}=R_{10}, R_{11}-1 \in E_{10}(5)$. So we assume $X_{D}$ is a 6 -distance set, and 6 distances are $D=d_{1}>d_{2}>d_{3}>d_{4}>d_{5}>d_{6}$. When $D G\left(X_{D}\right)=5 P_{2}$, we can prove $X_{D}=R_{12}-2$ (the 2 points absented has $D$-length) by the similar way as in the proof of Lemma 8 in [7]. When $D G\left(X_{D}\right)=P_{7} \cup P_{3}, P_{5} \cup P_{5}, P_{5} \cup P_{3} \cup P_{2}$ or $P_{4} \bigcup 2 P_{3}$, 
we have $X_{D}=R_{13}-3$ as shown in Figure $4 \mathrm{a}, 4 \mathrm{~b}, 4 \mathrm{~g}, 4 \mathrm{~h}$, respectively. However we omit proofs because if $\alpha\left(D G\left(X_{D}\right)\right)=6$, then we have $\alpha(D G(X))=6+4=10$, this implies $X$ contains $Y \in E_{10}(5)$. When $D G\left(X_{D}\right)=P_{4} \cup 3 P_{2}$, or $2 P_{3} \cup 2 P_{2}$, we can always find a contradiction, that is to say, these two types are not realizable for 6-distance, but we omit proofs of them because of the restriction of the length of the paper and the similar ways. In the following we depart five parts to prove the remain cases.

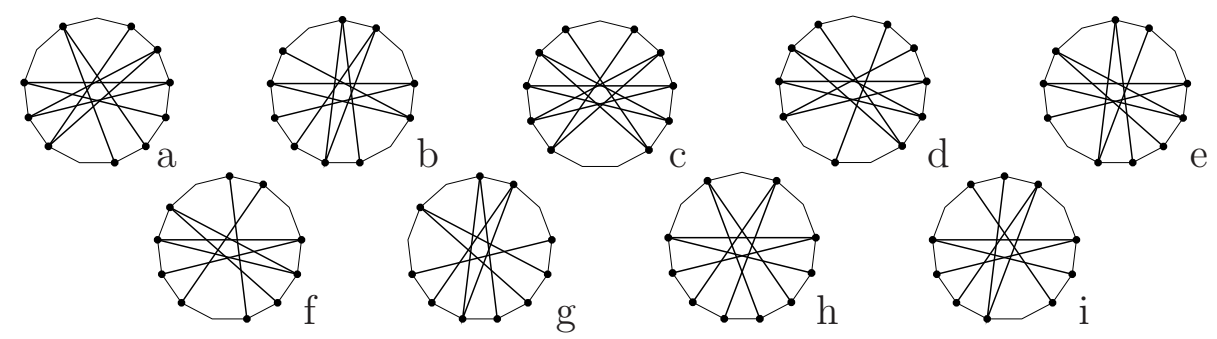

Figure 4: Dissimilar versions of $R_{13}-3$.

Part I: Suppose $2 P_{4} \cup P_{2}$, by Lemma 6 there exist two types:

Type one: we may denote $d(1,5)=d(1,6)=d(5,10)=d(4,9)=d(3,8)=d(4,8)=$ $d(2,7)=D$. Type two: denote $d(1,5)=d(1,6)=d(5,10)=d(4,9)=d(3,8)=d(3,7)=$ $d(2,7)=D$. We can prove type two is not realizable for 6 -distance, and the proof is omitted because of the restriction of the length of the paper. In the following we give the proof for type one by five steps. Clearly $d_{6} \leqslant d(i, i+1) \leqslant d_{5}$ for every $i \in X_{D}$.

Step I: Try to prove $d(9,10)=d_{6}$.

Suppose $d(9,10)=d_{5}$. By Lemma $7, d(1,9)=d(8,10)=d_{4}, d(1,8)=d(2,9)=$ $d(7,10)=d_{3}$ and $d(2,8)=d(3,9)=d(6,10)=d(1,7)=d_{2}$.

Assume $d(5,6)=d_{5}$. By Lemma $7, d(4,6)=d(5,7)=d_{4}, d(5,8)=d(4,7)=d(3,6)=$ $d_{3}$ and $d(2,6)=d(5,9)=d(3,7)=d_{2}$. Then we find points $5,6,9,10$ lie on a circle, points 1, 5, 7, 9 lie on a circle, points 5, 7, 8, 10 lie on a circle, points 1, 4, 6, 9 lie on a circle, points 1, 3, 6, 8 lie on a circle, points $2,3,6,9$ lie on a circle, points 2, 5, 8, 9 lie on a circle. If $d(2,3)=d_{5}$, then $d(2,5)=d_{3}$ and $d(2,4)=d(1,3)=d_{4}$, points $2,3,5,6$ lie on a circle, combining this with the former results we conclude all points of $X_{D}$ lie on a circle, then find all edges of $X_{D}$ have $d_{5}$-length, that is to say, no $d_{6}$-length appears. So $d(2,3)=d_{6}$. If $d(3,4)=d_{5}$, then $d(1,4)=d_{3}$ and $d(2,4)=d_{4}$, points $1,2,4,9$ lie on a circle, combining this with the former results we conclude all points of $X_{D}$ lie on a circle, then find $d_{5}=d(5,6)=d(2,3)=d_{6}$. So $d(3,4)=d_{6}$. If $d(8,9)=d_{5}$, then $d(6,9)=d_{3}$, points 5, 6, 8, 9 lie on a circle, combining this with the former results we conclude all points of $X_{D}$ lie on a circle, then find $d_{5}=d(8,9)=d(2,3)=d_{6}$. So $d(8,9)=d_{6}$. Now we can see points $2,3,4,8,9$ lie on a circle, and then all points of $X_{D}$ lie on a circle, which implies $d(1,10)=d_{6}$, but $d_{6}=d(1,10)=d(5,6)=d_{5}$, a contradiction. Hence we deduce $d(5,6)=d_{6}$, and similarly $d(3,4)=d_{6}$.

Assume $d(4,5)=d_{5}$. By Lemma $7, d(4,6)=d(3,5)=d_{4}, d(2,5)=d(4,7)=d(3,6)=$ $d_{3}$ and $d(2,6)=d(3,7)=d_{2}$. Then we find points $1,4,6,9$ lie on a circle, points $3,5,8$, 10 lie on a circle, points 4, 5, 9, 10 lie on a circle, points $3,4,5,6$ lie on a circle, points 2 , 
5, 7, 10 lie on a circle, points $2,3,6,9$ lie on a circle. If $d(2,3)=d_{5}$, then points $2,3,4$, 5 lie on a circle, combining this with the former results we conclude all points of $X_{D}$ lie on a circle, then find $d_{5}=d(2,3)=d(5,6)=d_{6}$. If $d(2,3)=d_{6}$, then points $2,3,5,6$ lie on a circle, combining this with the former results we conclude all points of $X_{D}$ lie on a circle, then find $d(8,9)=d(1,10)=d(7,8)=d_{6}$, and implies $d_{5} \leqslant d(2,4)=d(7,8)=d_{6}$, a contradiction. So $d(4,5)=d_{6}$.

Assume $d(6,7)=d_{5}$. By Lemma $7, d(6,8)=d(5,7)=d_{4}, d(5,8)=d(4,7)=d(6,9)=$ $d_{3}$ and $d(6,10)=d(5,9)=d(3,7)=d_{2}$. Then we find points $2,5,8,9$ lie on a circle, points $6,7,9,10$ lie on a circle, points $2,4,7,9$ lie on a circle, points 2, 3, 7, 8 lie on a circle, points $1,5,7,9$ lie on a circle, points $1,6,8,9$ lie on a circle. If $d(8,9)=d_{5}$, then $d(7,9)=d_{4}$, points $6,7,8,9$ lie on a circle, combining this with the former results we conclude all points of $X_{D}$ lie on a circle, then find $d_{5}=d(8,9)=d(3,4)=d_{6}$. If $d(8,9)=d_{6}$, then points $5,6,8,9$ lie on a circle, combining this with the former results we conclude all points of $X_{D}$ lie on a circle, then find $d_{5}=d(9,10)=d(5,6)=d_{6}$. Hence we deduce $d(6,7)=d_{6}$, and similarly $d(2,3)=d_{6}$.

We find $\angle 234>\angle 345$ since $d(5,8) \leqslant d_{3}$ and $d(2,8)=d_{2}$, and so $d(2,4)=d_{4}$, $d(3,5)=d_{5}$, similarly $d(5,7)=d_{4}, d(4,6)=d_{5}$. We find points $2,4,5,7$ lie on a circle, points 2, 5, 7, 10 lie on a circle, points 2, 4, 8, 10 lie on a circle, points 2, 5, 8, 9 lie on a circle, points 1, 2, 4, 9 lie on a circle, points 2, 3, 7, 8 lie on a circle, points 2, 3, 6, 7 lie on a circle, which imply all points of $X_{D}$ lie on a circle, and $d_{5}=d(9,10)=d(5,6)=d_{6}$, a contradiction.

Until now we conclude $d(9,10)=d_{6}$.

Step II: Try to prove $d(8,9)=d_{6}$ and $d(1,10)=d_{6}$.

Suppose $d(8,9)=d_{5}$. By Lemma $7, d(7,9)=d(8,10)=d_{4}, d(1,8)=d(6,9)=$ $d(7,10)=d_{3}$ and $d(2,8)=d(5,9)=d(6,10)=d(1,7)=d_{2}$.

Assume $d(3,4)=d_{5}$. By Lemma $7, d(2,4)=d(3,5)=d_{4}, d(2,5)=d(1,4)=d(3,6)=$ $d_{3}$ and $d(2,6)=d(4,10)=d(3,7)=d_{2}$. Then we find $d(2,3)=d_{6}$ since $\triangle 234 \cong \triangle * 98$ where $*=10$, points $2,4,7,9$ lie on a circle, points $2,4,8,10$ lie on a circle, points 2, 5, 7, 10 lie on a circle, points 1, 4, 6, 9 lie on a circle, points 1, 4, 7, 10 lie on a circle, points $3,6,7,10$ lie on a circle. If $d(6,7)=d_{5}$, then points $3,4,6,7$ lie on a circle, combining this with the former results we conclude all points of $X_{D}$ lie on a circle, and find $d_{5}=d(6,7)=d(2,3)=d_{6}$. If $d(6,7)=d_{6}$, then points $2,3,6,7$ lie on a circle, points $6,7,9,10$ lie on a circle, combining these with the former results we conclude all points of $X_{D}$ lie on a circle, then find all the remain edges have $d_{6}$-length, and $d_{1}=d(4,8)<d(4,9)=d_{1}$, a contradiction. Hence we deduce $d(3,4)=d_{6}$.

Assume $d(5,6)=d_{5}$. By Lemma $7, d(4,6)=d(5,7)=d_{4}, d(5,8)=d(4,7)=d(3,6)=$ $d_{3}$ and $d(5,9)=d(2,6)=d(3,7)=d_{2}$. Then we find points 5, 6, 8, 9 lie on a circle, points 1,3,6, 8 lie on a circle, points $3,6,7,10$ lie on a circle, points 5, 7, 8, 10 lie on a circle, points $2,3,7,8$ lie on a circle, points $4,6,7,9$ lie on a circle. If $d(6,7)=d_{5}$, then points $6,7,8,9$ lie on a circle, combining this with the former results we conclude all points of $X_{D}$ lie on a circle, then find $d_{5}=d(5,6)=d(9,10)=d_{6}$. So $d(6,7)=d_{6}$. If $d(2,3)=d_{6}$, then points $2,3,6,7$ lie on a circle, combining this with the former results we conclude all points of $X_{D}$ lie on a circle, then find $d_{5}=d(5,6)=d(9,10)=d_{6}$, a 
contradiction. If $d(2,3)=d_{5}$, then points $2,3,8,9$ lie on a circle, points $2,5,7,10$ lie on a circle, combining these with the former results we conclude all points of $X_{D}$ lie on a circle, then find $d_{5}=d(5,6)=d(9,10)=d_{6}$. So $d(5,6)=d_{6}$.

Assume $d(2,3)=d_{5}$. By Lemma $7, d(2,4)=d(1,3)=d_{4}, d(2,5)=d(3,10)=$ $d(1,4)=d_{3}$ and $d(4,10)=d(3,9)=d(2,6)=d_{2}$. Then we find points $3,4,9,10$ lie on a circle, points 5, 6, 9, 10 lie on a circle, points 2, 4, 7, 9 lie on a circle, points 2, 4, 8, 10 lie on a circle, points 2, 5, 6, 9 lie on a circle, points 3, 6, 9, 10 lie on a circle, points 1, 3, 7, 9 lie on a circle, and so conclude all points of $X_{D}$ lie on a circle, then find $d_{5}=d(8,9)=d(3,4)=d_{6}$, a contradiction. So $d(2,3)=d_{6}$.

Assume $d(1,10)=d_{5}$. By Lemma $7, d(2,10)=d(1,9)=d_{4}, d(2,9)=d(1,8)=$ $d(3,10)=d_{3}$ and $d(4,10)=d(3,9)=d_{2}$. Then we find points $2,3,4,5,6,7,9,10$ lie on a circle. If $d(1,4)=d_{3}$, then points $1,4,6,9$ lie on a circle, and so points $1,5,6,10$ lie on a circle, which implies $d_{5}=d(1,10)=d(5,6)=d_{6}$, a contradiction. If $d(1,4)=d_{4}$, then $d(2,4)=d_{5}$, points $1,2,4,10$ lie on a circle, and so points $1,5,6,10$ lie on a circle, which implies $d_{5}=d(1,10)=d(5,6)=d_{6}$, a contradiction. Hence we deduce $d(1,10)=d_{6}$.

Assume $d(4,5)=d_{5}$. By Lemma $7, d(4,6)=d(3,5)=d_{4}, d(2,5)=d(3,6)=d(4,7)=$ $d_{3}$ and $d(2,6)=d(3,7)=d_{2}$. Then we find points $1,5,6,9,10$ lie on a circle, points 4 , $6,7,9$ lie on a circle, points $2,3,7,8$ lie on a circle, points $2,3,4,5,6$ lie on a circle. If $d(6,7)=d_{6}$, then points $1,5,6,7,9,10$ lie on a circle, combining this with the former results we conclude all points of $X_{D}$ lie on a circle, then find $d_{6}=d(3,4)=d(8,9)=d_{5}$. If $d(6,7)=d_{5}$, then $d(5,7)=d(6,8)=d_{4}$, points $4,5,6,7$ lie on a circle, points $6,7,8$, 9 lie on a circle, combining these with the former results we conclude all points of $X_{D}$ lie on a circle, then find $d_{5}=d(8,9)=d(3,4)=d_{6}$, a contradiction. So $d(4,5)=d_{6}$.

We find $\angle 234>\angle 345$ since $d(5,8) \leqslant d_{3}$ and $d(2,8)=d_{2}$, and so $d(2,4)=d_{4}, d(3,5)=$ $d_{5}$, similarly $d(1,9)=d_{4}$. Now we find $d(1,4)=d(2,5)=d_{3}, d(4,10)=d(2,6)=d_{2}$, points 1, 4, 5, 6, 9, 10 lie on a circle, points 3, 4, 9, 10 lie on a circle, points 1, 4, 7, 10 lie on a circle, points 2, 4, 7, 9 lie on a circle, points 2, 4, 8, 10 lie on a circle, which imply all points of $X_{D}$ lie on a circle, and $d_{5}=d(8,9)=d(3,4)=d_{6}$, a contradiction.

Until now we conclude $d(8,9)=d_{6}$. And similarly $d(1,10)=d_{6}$.

Step III: Try to prove $d(5,6)=d_{6}$ and $d(3,4)=d_{6}$.

Suppose $d(5,6)=d_{5}$. By Lemma $7, d(5,7)=d(4,6)=d_{4}, d(5,8)=d(3,6)=d(4,7)=$ $d_{3}$ and $d(2,6)=d(5,9)=d(3,7)=d_{2}$.

Assume $d(2,3)=d_{5}$. By Lemma $7, d(2,4)=d(1,3)=d_{4}, d(2,5)=d(1,4)=$ $d(3,10)=d_{3}$ and $d(4,10)=d(3,9)=d_{2}$. Then we find points $2,3,5,6$ lie on a circle, points 2, 4, 5, 7 lie on a circle, points $3,4,7,10$ lie on a circle, points 4, 5, 9, 10 lie on a circle, points $3,5,8,10$ lie on a circle, points $1,4,5,8$ lie on a circle. If $d(3,4)=d_{5}$, then $d(3,5)=d_{4}$ and points $3,4,5,6$ lie on a circle, combining this with the former results we conclude all points of $X_{D}$ lie on a circle, but find $d_{5}=d(5,6)=d(1,10)=d_{6}$, a contradiction. If $d(3,4)=d_{6}$, then points $1,3,4,8,9,10$ lie on a circle, combining this with the former results we conclude all points of $X_{D}$ lie on a circle, but find $d_{5}=$ $d(5,6)=d(1,10)=d_{6}$, a contradiction. So $d(2,3)=d_{6}$.

Assume $d(1,2)=d_{5}$. By Lemma $7, d(2,10)=d(1,3)=d_{4}, d(2,9)=d(3,10)=d_{3}$ and $d(3,9)=d(2,8)=d(4,10)=d_{2}$. Then we find points $1,2,3,8,9,10$ lie on a circle, 
points 3, 5, 8, 10 lie on a circle, points 4, 5, 9, 10 lie on a circle, points 2, 3, 6, 9 lie on a circle, points $2,4,7,9$ lie on a circle, and so we conclude all points of $X_{D}$ lie on a circle, then find $d_{5}=d(5,6)=d(1,10)=d_{6}$, a contradiction. So $d(1,2)=d_{6}$.

We find $\angle 1 * 9>\angle 123$, where $*=10$, and so $d_{5} \leqslant d(1,3)<d(1,9) \leqslant d_{4}$, similarly $d(2,10)=d_{5}$. Now we find $d(1,8)=d(2,9)=d_{3}, d(3,9)=d(1,7)=d(2,8)=d_{2}$, points $1,2,8,9$ lie on a circle, points $1,2,3,10$ lie on a circle, points 2, 3, 8, 9 lie on a circle, which imply points $1,2,9,10$ lie on a circle, but now $d_{5}=d(2,10)=d(1,9)=d_{4}$, a contradiction.

Until now we conclude $d(5,6)=d_{6}$. And similarly $d(3,4)=d_{6}$.

Step IV: Try to prove $d(6,7)=d_{6}$ and $d(2,3)=d_{6}$.

Suppose $d(6,7)=d_{5}$. By Lemma $7, d(5,7)=d(6,8)=d_{4}, d(5,8)=d(6,9)=d(4,7)=$ $d_{3}$ and $d(6,10)=d(5,9)=d(3,7)=d_{2}$. Here points $1,5,6,8,9,10$ lie on a circle, points $3,4,8,9$ lie on a circle.

If $d(2,3)=d_{5}$, by Lemma $7, d(2,4)=d(1,3)=d_{4}, d(2,5)=d(1,4)=d(3,10)=d_{3}$ and $d(2,6)=d(4,10)=d(3,9)=d_{2}$. Then we find points $3,6,9,10$ lie on a circle, points 2, 3, 6, 7 lie on a circle, points 2, 5, 6, 9 lie on a circle, combining these with the former results we conclude all points of $X_{D}$ lie on a circle, and find $d(1,2)=d_{6}$, but $d_{2}=d(2,6)=d(1,5)=d_{1}$, a contradiction. So $d(2,3)=d_{6}$. Since points 1,8 , 9, 10 lie on a circle, we know $d(1,9)=d(8,10)$, and so $\angle 1 * 9=\angle 89 *$, where $*=10$, which imply $d(4,10)=d(5,9)=d_{2}$, points $4,5,9,10$ lie on a circle. Now we find $d(3,9)=d_{2}, d(3,10)=d_{3}$, points $3,4,7,10$ lie on a circle. For point 2, we have points 2, 4, 7, 8 lie on a circle, or points $2,3,8,9$ lie on a circle, since $d_{3} \leqslant d(2,8) \leqslant d_{2}$. Combining this with the former results we conclude all points of $X_{D}$ lie on a circle, but find $d_{3}=d(4,7) \neq d(3,10)=d_{3}$ since there must be $d(4,5)=d_{5}$, a contradiction.

Hence we deduce $d(6,7)=d_{6}$. And similarly $d(2,3)=d_{6}$.

Step V: Prove $X_{D}=R_{13}-3$ as shown in Figure 4i.

We have proved $d(1,10)=d(9,10)=d(8,9)=d(5,6)=d(6,7)=d(2,3)=d(3,4)=$ $d_{6}$. It is clear that at least one of remain edges of $X_{D}$ has $d_{5}$-length. If $d(1,2)=d_{5}$, by Lemma $7, d(2,10)=d(1,3)=d_{4}, d(2,9)=d(1,4)=d(3,10)=d_{3}$ and $d(2,8)=$ $d(4,10)=d(3,9)=d(6,10)=d_{2}$. Then we find points $1,2,3,4,8,9,10$ lie on a circle, points $1,5,6,10$ lie on a circle, points $4,5,9,10$ lie on a circle. If $d(7,8)=d_{6}$, then we find points $2,3,7,8$ lie on a circle, combining this with the former results we conclude all points of $X_{D}$ lie on a circle, but find $d_{2}=d(4,10) \neq d(5,9)=d_{2}$, a contradiction. So $d(7,8)=d_{5}$, points $1,2,7,8$ lie on a circle, and then all points of $X_{D}$ lie on a circle. Here $d(4,5)=d_{5}$, since otherwise $d_{1}=d(2,7) \neq d(3,8)=d_{1}$, a contradiction. In fact the remain three edges of $X_{D}$ must be $d_{5}$-length at the same time, that is to say, there must be $d(1,2)=d(4,5)=d(7,8)=d_{5}$. At last we obtain $X_{D}=R_{13}-3$ as shown in Figure 4 i.

Part II: Suppose $D G\left(X_{D}\right)=P_{10}$. By Lemma 6 we may denote $d(1,6)=d(1,7)=$ $d(2,7)=d(2,8)=d(3,8)=d(3,9)=d(4,9)=d(4,10)=d(5,10)=D$. By Lemma 7 , $d_{6} \leqslant d(i, i+1) \leqslant d_{5}$ for every $i \neq 10$.

Assume $d(7,8)=d_{5}$. Then by Lemma $7, d_{4}=d(6,8)<d(5,8)<d(4,8)<d(3,8)=D$ and $d_{4}=d(6,8)<d(6,9)<d(6,10)<d(1,6)=D$ and $d_{3}=d(6,9)<d(5,9)<d(4,9)=$ $D$. If $d(8,9)=d_{6}$, then $d_{3} \leqslant d(3,7)<d(2,9) \leqslant d_{2}$, and hence $d(3,7)=d_{3}$, clearly 
$d(3,4)=d(4,5)=d(5,6)=d(6,7)=d_{6}$. Now points $3,4,5,6,7$ lie on a circle, points 3, 4, 8, 9 lie on a circle, points 4, 5, 8, 9 lie on a circle. When $d(2,3)=d_{5}$, we can see points 2, 3, 7, 8 lie on a circle, and so conclude points 2, 3, 8, 9 lie on a circle, which implies $d_{5}=d(2,3)=d(8,9)=d_{6}$; when $d(2,3)=d_{6}$, we can see points $2,3,8,9$ lie on a circle, and so conclude points $2,3,7,8$ lie on a circle, which implies $d_{6}=d(2,3)=d(7,8)=d_{5}$, a contradiction. So $d(8,9)=d_{5}$. In fact $d(9,10)=d_{5}$, since otherwise $d(9,10)=d_{6}$ and $\angle 438<\angle 34 *<\frac{\pi}{2}$ where $*=10$, which can make $d_{2}=d(4,8)<d(3,10) \leqslant d_{2}$, a contradiction. Now by Lemma $7, d_{5}=d(9,10)<d(8,10)<d(7,10)=d_{3}$. Clearly $\triangle 678 \cong \triangle 789$ and $d(6,7)=d(7,8)=d_{5}$ since $d(6,8)=d(7,9)=d_{4}, d(7,8)=d(8,9)=d_{5}$ and $\angle 798=\angle 89 *-\angle 79 *=\angle 789-\angle 689=\angle 786$, where $*=10$. So points $6,7,8,9,10$ lie on a circle, points $5,7,8,10$ lie on a circle, points $2,3,7,9$ lie on a circle, points 3,4 , 8,10 lie on a circle, points $1,2,6,8$ lie on a circle. It is easy to see $\angle 789=\angle 89 *=\angle 678$, which implies $d(1,2)=d(2,3)=d(3,4) ; \angle 5 * 9=\angle 498$, which implies $d(4,5)=d(3,4)$. Hence points 1, 2, 3, 4, 5 lie on a circle, points 3, 5, 9, 10 lie on a circle. Until now we can see all points of $X_{D}$ lie on a circle, and $d(1,2)=d(2,3)=d(3,4)=d(4,5)=d(5,6)=d_{5}$ and $d(1,10)=d_{4}$. But we do not find $d_{6}$-length, a contradiction. So we can conclude $d(7,8)=d_{6}$, similarly $d(2,3)=d(3,4)=d(8,9)=d_{6}$, and points $2,3,4,7,8,9$ lie on a circle.

If $d(6,7)=d_{5}$, then $d_{3} \leqslant d(2,6)<d(1,8) \leqslant d_{2}$, and hence $d(2,6)=d_{3}$, clearly $d(2,3)=d(3,4)=d(4,5)=d(5,6)=d_{6}$, points $2,3,4,5,6$ lie on a circle. When $d(1,2)=d_{5}$, we can see points $1,2,6,7$ lie on a circle, and so conclude points 1,2 , 7,8 lie on a circle, which implies $d_{5}=d(1,2)=d(7,8)=d_{6}$; when $d(1,2)=d_{6}$, we can see points 1, 2, 7, 8 lie on a circle, and so conclude points 1, 2, 6, 7 lie on a circle, which implies $d_{6}=d(1,2)=d(6,7)=d_{5}$, a contradiction. So $d(6,7)=d_{6}$, similarly $d(9,10)=d(1,2)=d(4,5)=d_{6}$. At last we find all points of $X_{D}$ lie on a circle, and $d_{5} \leqslant d(5,6)<d(1,10) \leqslant d_{4}$, and $X_{D}=R_{13}-3$ as shown in Figure $4 \mathrm{c}$.

Part III: Suppose $D G\left(X_{D}\right)=P_{8} \cup P_{2}$. By Lemma 6 we may denote $d(1,6)=d(1,7)=$ $d(2,7)=d(2,8)=d(3,8)=d(3,9)=d(4,9)=d(5,10)=D$. By Lemma $7, d_{6} \leqslant$ $d(i, i+1) \leqslant d_{5}$ for every $i \in X_{D}$.

Assume $d(6,7)=d_{5}$. Then by Lemma $7, d_{4}=d(6,8)<d(6,9)<d(6,10)<d(1,6)=$ $D, d_{4}=d(5,7)<d(4,7)<d(3,7)<d(2,7)=D$ and $d_{4}=d(6,8)<d(5,8)<d(4,8)<$ $d(3,8)=D$ and $d_{3}=d(5,8)<d(5,9)<d(5,10)=D$. If $d(2,3)=d_{5}$, then by Lemma $7, d_{4}=d(2,4)<d(2,5)<d(2,6)<d(2,7)=D$ and $d_{4}=d(2,4)<d(1,4)<d(4,10)<$ $d(4,9)=D$ and $d_{3}=d(1,4)<d(1,5)<d(1,6)=D$, and points $2,3,6,7$ lie on a circle, points $2,4,5,7$ lie on a circle, points $2,4,6,8$ lie on a circle, points 2, 5, 6, 9 lie on a circle, points 4, 5, 9, 10 lie on a circle, and points $1,5,6,10$ lie on a circle. Then $d(7,8)=d_{5}$, since otherwise $d(7,8)=d_{6}$ and $\angle 128=\angle 127-\angle 827>\angle 217-\angle 617=\angle 216$, which can make $d_{2} \geqslant d(1,8)>d(2,6)=d_{2}$, a contradiction. Now points $2,3,7,8$ lie on a circle, and so all points of $X_{D}$ lie on a circle, we do not find $d_{6}$-length, a contradiction. So we can conclude $d(2,3)=d_{6}$. If $d(1,2)=d_{5}$, then by Lemma $7, d_{4}=d(1,3)<d(1,4)<d(1,5)<d(1,6)=$ $D$ and $d_{3}=d(1,4)<d(4,10)<d(4,9)=D, d_{4}=d(1,3)<d(3,10)<d(4,10)=d_{2}$, and points 1, 2, 6, 7 lie on a circle, points 1,3,6, 8 lie on a circle, points 1, 5, 6, 10 lie on a circle, points 3, 5, 8, 10 lie on a circle, and points 1, 4, 6, 9 lie on a circle. 
When $d(7,8)=d_{5}$, we can see points $1,2,6,7,8$ lie on a circle, and so conclude points $2,3,7,8$ lie on a circle, which implies $d_{6}=d(2,3)=d(7,8)=d_{5}$, a contradiction; when $d(7,8)=d_{6}$, we can see $d(1,8)<d(3,7)=d_{2}$ and $d(1,8)>d(2,6) \geqslant d_{3}$, a contradiction. So we can conclude $d(1,2)=d_{6}$. Now we can conclude $d(3,4)=d_{6}$, since otherwise $d_{2} \geqslant d(2,9)>d(4,8)=d_{2}$, a contradiction. Until now it must have $d(8,9)=d(7,8)$. If $d(7,8)=d(8,9)=d_{6}$, then points $1,2,3,4,7,8,9$ lie on a circle, and $d_{2}=d(1,8)>d(2,6) \geqslant d_{3}$, which imply $d(4,5)=d(5,6)=d_{6}$ and $2,3,4,5,6$ lie on a circle, so conclude points 1, 2, 6, 7 lie on a circle, which implies $d_{6}=d(1,2)=d(6,7)=d_{5}$, a contradiction. So we can conclude $d(7,8)=d(8,9)=d_{5}$. Then $d(4,5)=d_{6}$, since otherwise points $3,4,5,6,7,8,9$ lie on a circle, and $d_{6}=d(3,4)=d(8,9)=d_{5}$, a contradiction; and $d(5,6)=d_{6}$, since otherwise points $4,5,6,7,8,9$ lie on a circle, and $d_{6}=d(4,5)=d(7,8)=d_{5}$, a contradiction; and $d(9,10)=d_{6}$, since otherwise points 5 , $6,7,8,9,10$ lie on a circle, and $d_{6}=d(5,6)=d(9,10)=d_{5}$, a contradiction. Until now, no matter $d(1,10)=d_{6}$ or $d(1,10)=d_{5}$ we always find all points of $X_{D}$ lie on a circle, and so $d_{6}=d(1,2)=d(7,8)=d_{5}$, a contradiction. So finally we prove that $d(6,7)=d_{6}$.

And by the similarly way we can prove $d(3,4)=d(8,9)=d(7,8)=d(1,2)=d(2,3)=$ $d_{6}$. Then points $1,2,3,4,6,7,8,9$ lie on a circle.

Assume $d(9,10)=d_{6}$, then $\angle 89 *<\angle 234$ with $*=10$, which can make $d_{4} \geqslant d(2,4)>$ $d(8,10) \geqslant d_{5}$, and points $1,5,6,10$ lie on a circle, points $2,5,6,9$ lie on a circle, and so all points of $X_{D}$ lie on a circle, which implies $d_{5}=d(8,10)=d(2,4)=d_{4}$, a contradiction. So $d(9,10)=d_{5}$, and similarly $d(1,10)=d_{5}$.

When $d(5,6)=d_{5}$, points $1,5,6,9,10$ lie on a circle, and so all points of $X_{D}$ lie on a circle, which implies $d(4,5)=d_{6}$, obtain $X_{D}=R_{13}-3$ as shown in Figure $4 d$. When $d(5,6)=d_{6}$, we should consider the only case $d(4,5)=d_{6}$, and find all points of $X_{D}$ lie on a circle, clearly a contradiction exists.

Part IV: Suppose $D G\left(X_{D}\right)=P_{6} \cup P_{4}$. By Lemma 6 we may denote $d(1,6)=d(2,6)=$ $d(2,7)=d(3,7)=d(3,8)=d(4,10)=d(4,9)=d(5,10)=D$. By Lemma $7, d_{6} \leqslant$ $d(i, i+1) \leqslant d_{5}$ for every $i \neq 3$, and $d_{6} \leqslant d(3,4) \leqslant d_{4}$.

Assume $d(7,8)=d_{5}$. Then by Lemma $7, d_{4}=d(7,9)<d(7,10)<d(1,7)<d(2,7)=$ $D, d_{4}=d(6,8)<d(5,8)<d(4,8)<d(3,8)=D$ and $d_{4}=d(6,8)<d(6,9)<d(6,10)<$ $d(1,6)=D$ and $d_{3}=d(5,8)<d(5,9)<d(5,10)=D$. If $d(1,2)=d_{5}$, then $d(2,3)=d_{5}$, since otherwise $d_{2} \geqslant d(3,6)>d(1,7)=d_{2}$. At this time points $1,2,3,6,7,8$ lie on a circle, points 1, 3, 7, 9 lie on a circle, points 2, 6, 8, 10 lie on a circle, points 3, 4, 8, 9 lie on a circle, and points $1,5,6,10$ lie on a circle, and hence all points of $X_{D}$ lie on a circle, we do not find $d_{6}$-length, a contradiction. So $d(1,2)=d_{6}$. If $d(8,9)=d_{5}$, then $d(2,8)=d_{2}$ and $d(6,7)=d_{5}$, since otherwise $d_{2} \geqslant d(3,6)>d(2,8)=d_{2}$. Now we can see $d(3,6)=d_{2}$ and $d(2,3)=d_{6}$, points $6,7,8,9$ lie on a circle. Since $\triangle 368 \cong \triangle 48 *$ with $*=10$, clearly $\angle 94 *=\angle 84 *-\angle 849=\angle 638-\angle 637=\angle 738$, which can make $d(7,8)=d(9,10)=d_{5}$. Now points $6,7,8,9,10$ lie on a circle, points $1,6,8,9$ lie on a circle, points 1, 3, 6, 7 lie on a circle, points 2, 3, 6, 8 lie on a circle, and so points 2, 3, 6, 7 lie on a circle, which implies $d_{6}=d(2,3)=d(6,7)=d_{5}$, a contradiction. So $d(8,9)=d_{6}$. If $d(9,10)=d_{5}$, then $d(6,7)=d_{5}$. Now points $6,7,8,9,10$ lie on a circle, which implies $d_{6}=d(8,9)=d(6,7)=d_{5}$, a contradiction. So $d(9,10)=d_{6}$. Clearly we 
can see $d(4,5)=d(6,7)$, since $\angle 4 * 5=\angle 4 * 9-\angle 5 * 9=\angle 621-\angle 721=\angle 627$ with $*=10$. If $d(6,7)=d_{5}$, then points $4,5,6,7,8$ lie on a circle, points $4,6,7,9$ lie on a circle, and so points $4,5,8,9$ lie on a circle, which implies $d_{6}=d(8,9)=d(4,5)=d_{5}$, a contradiction. If $d(6,7)=d_{6}$, then points $4,5,6,7,8,9,10$ lie on a circle, and so $d(5,6)=d_{6}$. Hence $d_{3} \leqslant d(2,8)<d(3,6) \leqslant d_{2}$, and so $d(2,3)=d(1,2)=d_{6}$, and all points of $X_{D}$ lie on a circle, which implies $d_{6}=d(2,3)=d(7,8)=d_{5}$, a contradiction. So $d(7,8)=d_{6}$.

And by the similarly way we can prove $d(1,2)=d(2,3)=d(6,7)=d_{6}$. Then points $1,2,3,6,7,8$ lie on a circle.

Assume $d(9,10)=d_{5}$. Then by Lemma $7, d_{4}=d(1,9)<d(2,9)<d(3,9)<d(4,9)=$ $D, d_{4}=d(8,10)<d(7,10)<d(6,10)<d(5,10)=D$ and $d_{4}=d(8,10)<d(1,8)<$ $d(2,8)<d(3,8)=D$ and $d_{3}=d(1,8)<d(1,7)<d(1,6)=D$. If $d(4,5)=d_{5}$, then points 1, 4, 7, 8 lie on a circle, points 1, 2, 5, 8 lie on a circle, points 1, 5, 6, 10 lie on a circle, and points 4, 5, 9, 10 lie on a circle, and hence all points of $X_{D}$ lie on a circle, so $d(8,9)=d(1,10)=d_{6}$ and $d(5,6)=d_{5}$, which imply $d_{5}=d(4,5)=d(6,7)=d_{6}$, a contradiction. So $d(4,5)=d_{6}$, and $d_{2}=d(1,7)>d(5,9) \geqslant d_{3}$, which can make $d(5,6)=$ $d(8,9)=d_{6}$ and points $1,2,3,5,6,7,8,9$ lie on a circle, now $D=d(2,6)=d(1,5) \leqslant d_{2}$, a contradiction. So $d(9,10)=d_{6}$. Similarly $d(4,5)=d_{6}$, and hence points $4,5,9$, 10 lie on a circle.

Assume $d(8,9)=d_{5}$. Then points $6,7,8,9,10$ lie on a circle, and hence points 1 , $2,3,6,7,8,9,10$ lie on a circle, which imply $d(1,10)=d_{6}$ and $d(2,10)=d(1,9)$. But $\angle 21 * \neq \angle 1 * 9$ with $*=10$, a contradiction. So $d(8,9)=d_{6}$.

If $d(3,6)=d(1,7)=d(2,8)=d(5,9)=d_{3}$, then $d(1,10)=d(5,6)=d_{6}$, all points of $X_{D}$ lie on a circle, which imply $d_{1}=d(2,6)=d(1,5) \leqslant d_{2}$, a contradiction. So $d(3,6)=$ $d(1,7)=d(2,8)=d(5,9)=d_{2}$, and hence $d(3,9)=d(1,5)=d(4,8)=d_{2}$, all points of $X_{D}$ lie on a circle. Now $d(5,6)=d_{5}$ and $d(1,10)=d_{5}$, since $d_{6}=d(1,2) \neq d(5,6) \leqslant d_{5}$ and $d_{6}=d(4,5) \neq d(1,10) \leqslant d_{5}$. Clearly we can see now it must have $d(3,4)=d_{5}$, and obtain $X_{D}=R_{13}-3$ as shown in Figure 4 e.

Part V: Suppose $D G\left(X_{D}\right)=P_{6} \cup 2 P_{2}$. By Lemma 6 we may denote $d(1,6)=d(2,6)=$ $d(2,7)=d(3,7)=d(3,8)=d(4,9)=d(5,10)=D$. By Lemma $7, d_{6} \leqslant d(i, i+1) \leqslant d_{5}$ for every $i \in X_{D}$.

Assume $d(7,8)=d_{5}$. Then by Lemma $7, d_{4}=d(7,9)<d(7,10)<d(1,7)<d(2,7)=$ $D, d_{4}=d(6,8)<d(5,8)<d(4,8)<d(3,8)=D$ and $d_{4}=d(6,8)<d(6,9)<d(6,10)<$ $d(1,6)=D$ and $d_{3}=d(5,8)<d(5,9)<d(5,10)=D$. If $d(1,2)=d_{5}$, then $d(2,3)=d_{5}$, since otherwise $d_{2} \geqslant d(3,6)>d(1,7)=d_{2}$. At this time points $1,2,3,6,7,8$ lie on a circle, points 1, 3, 7, 9 lie on a circle, points 2, 6, 8, 10 lie on a circle, points 3, 4, 8, 9 lie on a circle, and points 1, 5, 6, 10 lie on a circle, and hence all points of $X_{D}$ lie on a circle, no $d_{6}$-length exists, a contradiction. So $d(1,2)=d_{6}$. If $d(8,9)=d_{5}$, then $d(2,8)=d_{2}$ and $d(6,7)=d_{5}$, since otherwise $d_{2} \geqslant d(3,6)>d(2,8)=d_{2}$. Now we can see $d(3,6)=d_{2}$ and $d(2,3)=d_{6}$. If $d(1,10)=d_{5}$, then points $1,6,7,8,9$, 10 lie on a circle, which implies $d(9,10)=d_{5}$ and $d(1,9)=d(7,9)=d_{4}, \angle 1 * 9=$ $\angle 987$ with $*=10$. But in fact $\angle 1 * 9=\angle 1 * 5+\angle 5 * 9<\angle 783+\angle 389=\angle 789$, a contradiction. So $d(1,10)=d_{6}$. If $d(9,10)=d_{5}$, then $\triangle 89 * \cong \triangle 789$ and $\angle 789=\angle 89 *$, 
but $\angle 789=\angle 783+\angle 389 \neq \angle 49 *+\angle 894=\angle 89 *$, a contradiction. So $d(9,10)=d_{6}$. Then $\angle 123>\angle 1 * 9$, and so $d_{4} \geqslant d(1,3)>d(1,9) \geqslant d_{5}$, and points $1,3,6,7$, 8 lie on a circle, points 2, 3, 6, 8 lie on a circle, which make points $2,3,6,7$ lie on a circle, and $d_{6}=d(2,3)=d(6,7)=d_{5}$, a contradiction. So $d(8,9)=d_{6}$. If $d(9,10)=d_{5}$, then $d(6,7)=d_{5}$ and $d(3,6)=d(2,8)=d_{2}$, since otherwise $d_{2} \geqslant d(3,6)>d(2,8)=d_{2}$. Now points $6,7,8,9,10$ lie on a circle, which implies $d_{6}=d(8,9)=d(6,7)=d_{5}$, a contradiction. So $d(9,10)=d_{6}$. If $d(1,10)=d_{5}$, then $d(6,7)=d_{5}$ and points $1,2,8$, 9, 10 lie on a circle, points 1, 6, 7, 8, 10 lie on a circle, which imply points 1, 2, 6, 7 lie on a circle, $d_{6}=d(1,2)=d(6,7)=d_{5}$, a contradiction. So $d(1,10)=d_{6}$. Now we have $\angle 21 *>\angle 1 * 9=\angle * 98$, and hence $d(2,10)=d_{4}$ and $d(1,9)=d(8,10)=d_{5}$. Then we can find $d(2,3)=d_{6}$, since $\angle 3 * 2=\angle 6 * 2-\angle 6 * 3=\angle 368-\angle 369=\angle 968$, and $\triangle 3 * 2 \cong \triangle 968$. Finally we find points $1,2,9,10$ lie on a circle, and so $d_{4}=d(2,10)=d(1,9)=d_{5}$, a contradiction. So at last we obtain $d(7,8)=d_{6}$.

And by the similarly way we can prove $d(1,2)=d(2,3)=d(6,7)=d_{6}$. Then points $1,2,3,6,7,8$ lie on a circle.

Assume $d(8,9)=d_{5}$. Then by Lemma $7, d_{4}=d(7,9)<d(6,9)<d(5,9)<d(4,9)=$ $D, d_{4}=d(8,10)<d(1,8)<d(2,8)<d(3,8)=D$ and $d_{4}=d(7,9)<d(7,10)<d(1,7)<$ $d(2,7)=D$ and $d_{3}=d(6,9)<d(6,10)<d(1,6)=D$. If $d(9,10)=d_{6}$, then points $1,2,3,6,7,8,9,10$ lie on a circle, which makes $d(1,10)=d_{6}$ and $d(1,3)=d(1,9)$, which contradicts the fact $\angle 123 \neq \angle 1 * 9$. So $d(9,10)=d_{5}$. Then $\triangle 829 \cong \triangle * 69$ and $\angle 829=\angle * 69$, and $\angle 26 *=\angle 269-\angle 96 *=\angle 629-\angle 829=\angle 628$, and so $d(1,10)=d_{6}$, points $1,2,3,6,7,8,10$ lie on a circle. If $d(5,6)=d_{6}$, then points $1,5,6,10$ lie on a circle, and so points $1,2,5,6$ lie on a circle, which imply $d_{2} \geqslant d(1,5)=d(2,6)=d_{1}$, this is impossible. If $d(5,6)=d_{5}$, then points $5,6,8,9$ lie on a circle, points $2,5,8,9$ lie on a circle, and so points $6,7,9,10$ lie on a circle, $d_{6}=d(6,7)=d(9,10)=d_{5}$, this is impossible. So at last we obtain $d(8,9)=d_{6}$. Similarly $d(1,10)=d_{6}$.

Assume $d(4,5)=d_{5}$. Then by Lemma $7, d_{4}=d(3,5)<d(2,5)<d(1,5)<d(5,10)=$ $D, d_{4}=d(4,6)<d(4,7)<d(4,8)<d(4,9)=D$. If $d(9,10)=d_{5}$, then we can see all points of $X_{D}$ lie on a circle, and so $d(5,6)=d(3,4)=d_{5}$, obtain a contradiction $d_{4}=d(3,5) \neq d(1,9)=d_{4}$. So $d(9,10)=d_{6}$. We know $d_{3} \leqslant d(1,7) \leqslant d_{2}$. If $d(1,7)=d_{3}$, then points $1,7,8,9$, 10 lie on a circle, points $3,4,7,9$ lie on a circle, points 2, 5, 6, 10 lie on a circle, and so we can see all points of $X_{D}$ lie on a circle, which implies $d(5,6)=d_{6}$, obtain a contradiction $d_{2}=d(1,5)=d(2,6)=d_{1}$. If $d(1,7)=d_{2}$, then $d(3,6)=d(2,8)=d_{2}, d(1,9)=d(8,10)=d_{5}$, and hence can obtain points $1,2,9$, 10 lie on a circle, so $d(2,10)=d(1,9)$, which contradicts the fact that $d(2,10) \neq d(1,9)$ because of $\angle 21 * \neq \angle 1 * 9$, where $*=10$. So $d(4,5)=d_{6}$.

Assume $d(9,10)=d_{6}$. Recall that points $1,2,3,6,7,8$ lie on a circle. If $d(1,7)=d_{3}$ or $d(2,8)=d_{3}$ or $d(6,10)=d_{3}$ or $d(3,9)=d_{3}$, then points $1,2,3,6,7,8,9,10$ lie on a circle. Clearly $d(5,6)=d_{5}$, since otherwise points $1,5,6,10$ lie on a circle, and so points $1,2,5$, 6 lie on a circle, there exists a contradiction $d_{2} \geqslant d(1,5)=d(2,6)=d_{1}$. Correspondingly $d(3,4)=d_{5}$, since otherwise points $3,4,8,9$ lie on a circle, and so points $3,4,7,8$ lie on a circle, there exists a contradiction $d_{2} \geqslant d(4,8)=d(3,7)=d_{1}$. Until now we can see all points of $X_{D}$ lie on a circle, and obtain a contradiction $D=d(3,8) \neq d(5,10)=D$. 
So it must have $d(1,7)=d(2,8)=d(6,10)=d(3,9)=d_{2}$, then points $1,6,7,10$ lie on a circle, points $2,3,8,9$ lie on a circle, and so conclude points 1, 2, 9, 10 lie on a circle, $d(2,10)=d(1,9)$, contradicts the fact $\angle 21 * \neq \angle 1 * 9$. So $d(9,10)=d_{5}$.

Recall that we have prove that $d(7,8)=d(1,2)=d(2,3)=d(6,7)=d(8,9)=$ $d(1,10)=d(4,5)=d_{6}$ and $d(9,10)=d_{5}$, points $1,2,3,6,7,8,9,10$ lie on a circle. We can deduce $d(3,4)=d_{5}$, since otherwise points $3,4,8,9$ lie on a circle, and so points 3,4 , 7, 8 lie on a circle, obtains a contradiction $d_{2} \geqslant d(4,8)=d(3,7)=d_{1}$; and $d(5,6)=d_{5}$, since otherwise points 1, 5, 6, 10 lie on a circle, and so points 1, 2, 5, 6 lie on a circle, obtains a contradiction $d_{2} \geqslant d(1,5)=d(2,6)=d_{1}$. Now all points of $X_{D}$ lie on a circle, we obtain $X_{D}=R_{13}-3$ as shown in Figure 4 f.

Theorem 17. No 14-point 6-distance set with $m=\left|X_{D}\right|=10$ exists.

Proof. Let $X$ be a 14-point 6-distance set with $m=\left|X_{D}\right|=10$. By Theorem 16, $X$ contains a subset $Y \in\left\{R_{12}-2, R_{13}-3\right\} \cup E_{10}(5)$. Clearly adding points to them such that the total number to be 14 force a seventh distance, a contradiction to 6 -distance.

\section{Maximum planar 6-distance set}

Theorem 18. There exists no 14-point 6-distance set in the plane.

Proof. Let $X$ be a 14-point 6-distance set. If $m \geqslant 12$, then by Lemma 3 and Lemma 4 , the subset $X_{D} \subset X$ is a convex $m$-gon, and $X$ contains a subset $Y \in \bigcup_{k \leqslant 6} M_{12}(k)=$ $\left\{R_{12}, R_{13}-1\right\}$. For $m=10,11$, we have given the proof as before. If $m=9$, and if $D G\left(X_{D}\right)=C_{9}$, then $X_{D}=R_{9}$ as showed in [9]; and if $D G\left(X_{D}\right) \neq C_{9}$, by Lemma 9, $\alpha\left(D G\left(X_{D}\right)\right) \geqslant 5, X$ contains a subset $Y \in E_{10}(5)$ by removing four points from $X_{D}$ with the diameter $D$ been eliminated. If $m \leqslant 8$, then $14-\left\lceil\frac{m}{2}\right\rceil \geqslant 10$, and by Lemma $3, X$ contains a subset $Y \in E_{10}(5)$. But adding points to $R_{9}, R_{12}, R_{13}-1$ or $Y \in E_{10}(5)$ such that the total number of points being 14 always force a seventh distance.

Recall that $g(6) \geqslant 13$, since $R_{13}, R_{12}^{+}$and the only configuration in $L_{\triangle}$ as shown in Figure 1 are known 13-point 6-distance sets. Until now we can conclude that:

Theorem 19. $g(6)=13$, that is, 13 is the maximum number of points in the plane that determine exactly 6 different distances.

\section{Acknowledgements}

Thanks to the referee for suggesting the proof of Theorem 16.

\section{References}

[1] P. Erdős and P. Fishburn. Maximum planar sets that determine k distances. Discrete Mathematics, 160:115-125, 1996. 
[2] E. Altman. On a problem of P. Erdős. American Mathematical Monthly, 70:148-157, 1963.

[3] P. Erdős and P. Fishburn. Convex nonagons with five intervertex distance. Geometria Dedicata, 60:317-332, 1996.

[4] P. Fishburn. Convex polygons with few intervertex distance. Computational Geometry, 5:65-93, 1995.

[5] M. Shinohara. Uniqueness of maximum planar five-distance sets. Discrete mathematics, 308:3048-3055, 2008.

[6] M. Shinohara. Classification of three-distance sets in two dimensional Euclidean space. European Journal of Combinatorics, 25:1039-1058, 2004.

[7] X. Wei. Classification of eleven-point five-distance sets in the plane. Ars Combinatoria, 102:505-515, 2011.

[8] W. Lan and X. Wei. Classification of four-distance seven-point sets in the plane. Mathematical Notes, 2013. to appear.

[9] X. Wei. Distance sets with diameter graph being cycle. submitted and revised. 